\title{
Chemists: Al Is Here; Unite To Get the Benefits
}

DOI:

10.1021/acs.jmedchem.0c00163

\section{Document Version}

Accepted author manuscript

Link to publication record in Manchester Research Explorer

\section{Citation for published version (APA):}

Griffen, E. J., Dossetter, A. G., \& Leach, A. G. (2020). Chemists: Al Is Here; Unite To Get the Benefits. Journal of Medicinal Chemistry. https://doi.org/10.1021/acs.jmedchem.0c00163

\section{Published in:}

Journal of Medicinal Chemistry

\section{Citing this paper}

Please note that where the full-text provided on Manchester Research Explorer is the Author Accepted Manuscript or Proof version this may differ from the final Published version. If citing, it is advised that you check and use the publisher's definitive version.

\section{General rights}

Copyright and moral rights for the publications made accessible in the Research Explorer are retained by the authors and/or other copyright owners and it is a condition of accessing publications that users recognise and abide by the legal requirements associated with these rights.

\section{Takedown policy}

If you believe that this document breaches copyright please refer to the University of Manchester's Takedown Procedures [http://man.ac.uk/04Y6Bo] or contact uml.scholarlycommunications@manchester.ac.uk providing relevant details, so we can investigate your claim.

\section{OPEN ACCESS}




\title{
Chemists: AI is here, unite to get the benefits
}

\author{
Edward J. Griffen*, Alexander G. Dossetter, Andrew G. Leach \\ MedChemica Ltd, Alderley Park, Macclesfield, Cheshire. SK10 4TG
}

Keywords:

Artificial Intelligence, medicinal chemistry, computational chemistry, interdisciplinary behaviors.

Abstract:

The latest developments in artificial intelligence(AI) have arrived into an existing state of creative tension between computational and medicinal chemists. At their most productive, medicinal and computational chemists have made significant progress in delivering new therapeutic agents into the clinic. However, the relationship between these communities has the prospect of being weakened by application of over-simplistic AI methods which, if they fail to deliver, will reinforce unproductive prejudices. We review what can be learnt from our history of integrating QSAR and structure-based methods into drug discovery. Now with synthesis and testing available as contract services, the environment for computational innovation has changed and we consider the impact this may have on the relationships in our disciplines. We discuss the current state of interdisciplinary communication and suggest approaches to bring the sub-disciplines together in order to improve computational medicinal chemistry and, most importantly, deliver better medicines to the clinic faster. 


\section{What is Artificial intelligence in Medicinal Chemistry?}

To refer to Artificial intelligence(AI) immediately requires clarification because of the age of the term and its checkered history. Here we will take the approach suggested by Edward Feigenbaum ${ }^{1}$ in suggesting that there is intelligence when a system when presented with a problem generates a solution indistinguishable from that generated by a skilled human. This is like the Turing test ${ }^{2}$ but just applied to a specific field. The usefulness of this definition is that is focusses on outputs rather than the mechanics of a system. If we then consider intelligence there are six facets we can describe. The first three are: the synthesis of information, pattern recognition and decision making. The second three are all based around autonomy: intelligence reacts to information and based on some goals, generates a proposal to increase the likelihood of achieving that goal, it makes mistakes, but then learns from them, and at the most sophisticated level exhibits strategic thinking and situational awareness to avoid being trapped in local minima. ${ }^{3}$ This draws the distinction with machine learning which is just a set of statistical methods used for either labelling sub sets of a data set, or generating a relationship between a measurable property and some descriptors. One of the areas of confusion is that, early in the study of artificial intelligence, computational models were built which were designed by analogy to neurological structures. Neural networks and their progenitor, the perceptron ${ }^{4}$ were inspired by biological systems but are just varieties of non-linear model fitting or classification methods. Some authors therefore consider the use of a neural network method of machine learning to be sufficient to designate work 'artificial intelligence', however here we refer particularly to the more demanding definition above.

Within the medicinal chemistry context examples of the first three aspects of artificial intelligence could be the compilation of chemical structures and biological data, the generation of a (Q)SAR 
model and the ability to prioritise suggested compounds based on a model. The second three of these could be a system that monitors in vitro clearance data being registered to a database and thenproposes new compounds for synthesis to decrease metabolism with an understanding that low metabolism is a project goal. By also updating the local model for metabolism if the previous round of compounds synthesised have not achieved their goal, a new round of compounds can be proposed based on the updated model. Finally, in addressing a goal the system could have a hierarchy of global and local models and understand that when the current local model of clearance is insufficiently powered it would lead to riskier proposed compounds than a global model which might be based on a far larger data set. Equally artificial intelligence in chemistry encompasses systems that generate synthetic routes, assess the probability of reaction success and side products, or predict crystalline forms. The essential feature is the automated compilation and analysis of data to generate 'human like' suggestions to solve problems.

\section{The current state of the drug hunting ecosystem}

The current generation of AI approaches for medicinal chemistry has arrived into a context of consistent decline in drug hunting productivity. ${ }^{5}$ This has been attributed to a number of factors, but the simplest explanation is the "low hanging fruit" argument - that the current biological targets for drug hunting are intrinsically harder than those previously addressed. ${ }^{6}$

The headcount reductions in large Pharma over the last decade and outsourcing of synthetic and medicinal chemistry to contract research organizations (CROs) has been a cost cutting response to the productivity crisis but efficiency gains have their limits. The arrival of a new generation of machine learning methods has been heralded by many as the cavalry coming to save drug hunting. 
By others it has been viewed as the arrival of another "distraction" from the "proper" work of making and testing compounds, improving biological activity and solving both ADMET and physicochemical problems. Some have viewed this as the next generation of efforts by computational chemists who have previously contributed QSAR and structure-based drug design, which were equally heralded as universal solutions to medicinal chemistry problems. ${ }^{7}$ The difference now is that whereas in the past large scale capital infrastructure was needed for both significant computational efforts as well as synthetic chemistry, CROs have made compound acquisition a contract resource rather than a long term investment. Similarly, the availability of cloud computing means that anyone with a credit card and enough computer science expertise can create a high-performance computing cluster and conduct large scale calculations without capital expenditure, hardware or significant delay. A recent iteration of this is "virtual screening as a service" where it is possible to screen and filter a massive library of virtual molecules on cloud infrastructure that synthetic chemistry houses have high confidence in being able to make, all as a contract service. ${ }^{89}$ This commodification of lead identification clearly shows the direction the field is moving in. Even in the lead optimization phase, many companies are moving increasingly to software-as-a-service, ${ }^{10-13}$ with capabilities that previously would have required a specialist workstation now being delivered to a web browser. In parallel, there has been a rise in the number of Virtual Pharma where all the experimental resources are externalized and the core team acts as an "air traffic control group" making sure the right work is done in the right sequence in the right places. From an investment perspective this makes complete sense as all the "turn-key" resources can be focused on generating a clinical asset, leaving little to no residual assets to dispose of once the clinical compound is licensed. 
There is nothing intrinsically wrong in this model, in fact the "niche specialist strategy" where experts in particular sub-disciplines of drug hunting do more of what they're good at should drive up the quality of work, especially if they can retain expertise within CROs. However, it does enable some counter-productive possibilities. An under-discussed issue in the chemistry domain is the relationship between medicinal and computational chemists. As members of one subdomain who have migrated to the interdisciplinary border between the two we believe we have some useful observations for mutual benefit.

\section{The medicinal - computational chemist relationship}

Historically, many of these intra-disciplinary relationships were hidden in large Pharma silos and only rarely explicitly discussed. ${ }^{14,15}$ A frequent model in Pharma or CROs is to have a computational chemist advising several projects, reviewing and recommending compounds for synthesis but without decision making authority or accountability for progress. The medicinal chemist with the accountability for progress would, in an ideal world, use all the human and computational resources to make the best decisions on the compounds to make and drive the project forward. Clearly this is painted as an ideal view and in many cases this high-performing team model has worked well with creativity from all parties; the combination of thoughtful challenge from computational chemists with synthetic pragmatism from medicinal chemists has been highly productive. However, underlying the relationship are some truths that the current AI generation is bringing to the surface challenging the positive spirit of collaboration. 
Medicinal and computational chemists each come from different backgrounds in both experience and world view. One feature of working with both medicinal and computational chemists is that the effort and duration of training combined with the small number of these professionals, means that as is common to other highly skilled, small groups, designation of what kind of chemist they are frequently becomes a strong part of people's personal identity. This is commonly heard as people introduce themselves with "I am a medicinal/computational chemist" rather than "I do medicinal/computational chemistry". In their early industrial training, both parties often need to gain an appreciation of the skills and attributes of the other. Most medicinal chemists come from a synthetic chemistry background, which is unusual in the sciences as it is predominantly a constructive rather than a deductive activity. Often the only hypothesis a synthetic chemist is testing is "will this combination of reagents lead to the desired product". In their academic lives many synthetic chemist's progress and success are measured and rewarded in terms of number of successful steps in a synthetic route achieved, or yields improved in method development. Synthetic chemists are hailed for work rate and tenacity - both essential attributes in drug hunting where the success rate in generating acceptable molecules is notoriously low. The requirement to handle dangerous reagents also plays to a certain machismo and the field has its own share of horror stories of poor management behavior. Conversely, those drawn to computational chemistry have prioritized understanding over output, and may have preferred to avoid the synthetic laboratory for reasons of safety and culture. A cartoon view is of people looking for an elegant "theory of everything" that will be universally applicable. There are two common features that the training of both computational chemists and synthetic chemists share that can disable rather than enable a good working relationship. Firstly, neither group deals with measurements with significant variance. Synthetic chemists make analytical measurements to 
determine the structures of their compounds, these analytical methods have been optimized to have low variance so that they can also be used as evidence of purity. Computational chemists almost always are consumers of data rather than producers and only rarely, in the authors experience, have a strong appreciation of the intrinsic variability of measurement systems. One part of a chemist's induction into drug hunting is to gain the understanding that biological measurements are intrinsically variable because of their complexity and sensitivity to conditions and that results will show a significant range. Though both medicinal and computational chemists may understand this, they often have a "blind spot" when it comes to computational models in expecting them to be more accurate than the biological system they are measuring. Clearly when aiming to make predictions in an assay which shows a standard deviation of 0.15 pIC50 units, (meaning 95\% of measurements will be within 0.3 , or 2 fold of the 'true' value - a good assay) it is ridiculous to suggest that a prediction is accurate within 0.2 pIC50 units. There has been significant progress in the field in describing this problem in the last decade. ${ }^{16-19}$ However an appreciation of the intrinsic limits of modelling biological systems seems to be difficult, this may be because the computational chemist having a sense of professional pride, feels that they should do better - and are therefore embarrassed by the quality of prediction, and the medicinal chemist hearing the message from a 'fellow chemist' is expecting to hear more precise predictions, whereas if the modelling was provided by a biologist they might be attuned to accepting the variance more easily. There is a need for computational chemists to well delineate the limits of their techniques and set expectations reasonably, and equally for medicinal chemists to both appreciate the bounds of what is on offer and also recognize that an equivalent evaluation of their own "hunches and predictions' might not perform any better. Attempts to enquire into the mind of medicinal chemists have shown them to be all too human in their inconsistency and biases. ${ }^{20}$ This is an excellent example of the 
all too familiar issue of the espoused working practice not matching the practice in use. ${ }^{21}$ Secondly, in the academic careers of both synthetic and computational chemists work is predominantly carried out on solitary projects rather than in teams. The result of this is that the people that are most successful are very determined but have often worked mainly as individuals. This means that the pool of talent to be recruited from have not needed to spend much time developing their team working skills. In particular, these manifest as behaviors such as showing unilateral control, a win-lose approach to conflict management and suppression of personal doubt. Most experienced chemists in both fields know of the dysfunctional relationships that this lead to: a medicinal chemist acts as a hard gatekeeper and exploits that positional power to drive the project in their chosen direction ignoring the well-founded hypotheses and data brought by the computational chemist because it does not fit with the medicinal chemist's world view. Quite rationally, in response and frustration, the computational chemist may tactically withdraw from such a situation and focus their efforts where their input is more valued. As medicinal chemists can be accused of ignoring their computational chemistry colleagues, the parallel behavior of some, often academic, computational chemists equally occurs. This response is to refine models by optimizing algorithms or descriptors where resources would have been better spent generating more compounds and data. Both of these behaviors stem from the same core problem: when challenged with either a hypothesis that is in conflict with their world view or the risk of generating data that might upset a beautiful hypothesis, both parties adopt a defensive strategy. For most people avoiding embarrassment is a strong drive. For highly trained individuals where much of their identity is associated with their professional standing, avoiding embarrassment due to failure is one of the most potent motivators - and this pushes us into defensive strategies. As Argyris recognized: "whenever human beings are faced with any issue that contains significant 
embarrassment or threat, they act in ways that bypass, as best they can, the embarrassment or threat. In order for the bypass to work, it must be covered up ... Organizational defensive routines are actions or policies that prevent individuals or segments of the organization from experiencing embarrassment or threat. Simultaneously, they prevent people from identifying and getting rid of the causes of the potential embarrassment or threat. Organizational defensive routines are antilearning, overprotective, and self-sealing." ${ }^{22}$ Alas, drug hunting is truly a domain where "no plan survives first contact with the enemy"23 and failure is the norm rather than an exception which inevitably leads to very significant pressure. This places both medicinal and computational chemists at high risk of adopting defensive strategies both individually and within organizational groups. When working effectively together, both sides in this alliance have played to each others strengths and delivered compounds into the clinic. In biotech companies and small drug discovery units we have worked with in the last decade, a new drug hunting group always wants both a medicinal and a computational chemist to start with. The medicinal chemist to be responsible for the choice of compounds and supervise synthesis, and a computational chemist to generate 3D models, examine structural data and run advanced calculations. In small biotech companies, the shared goals of survival and generating salable assets can pull a team together and overcome the bias to defensive behavior. To build good AI systems in chemistry however, both medicinal and computational chemists are going to have to escape from the organizational defense strategies that allow them to avoid change and to instead embrace each other's approaches. ${ }^{21}$

Whatever our position on the approaches to integrating computational chemistry into drug discovery, recent analysis of the sources of candidate drugs points to their essential value. ${ }^{24}$ (Figure 1) With a quarter of candidate drugs disclosed in J. Med. Chem. between 2016 and 2017 being derived by either structure-based drug design, fragment screening or directed screening, as a bare 
minimum it is incontrovertible that these projects required the use of computational methods. In reality, the optimization of random screen-derived compounds or those optimized from a known drug will also have used computational tools in a significant number of cases.

\section{Lead Generation Strategy (\%)}

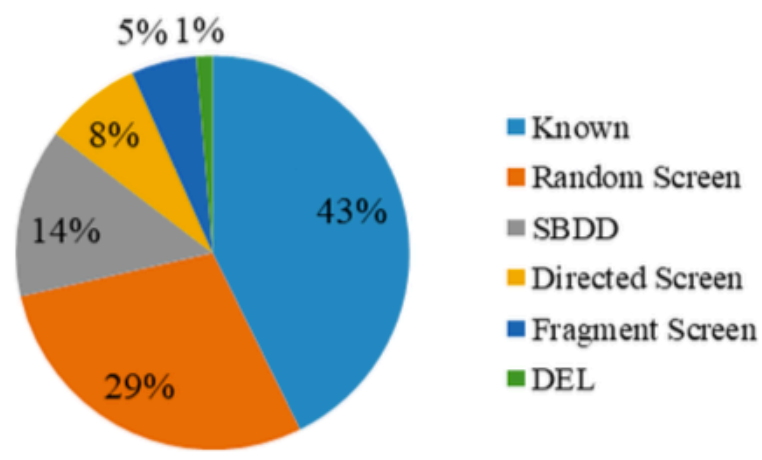

Figure 1. Distributions of the six sources of lead generation strategies: Known compounds, Random screen or high throughput screen (HTS), Fragment screen or Fragment based lead generation (FBLG), DNA Encoded Libraries (DEL), Structure based drug design (SBDD), Directed Screen for the 66 hit-to-candidate pairs in $J$. Med. Chem. 2016-2017.

Figure 1

From Brown and Bostrom ${ }^{24}$

\section{What can past experience teach us?}

What of history? As we have alluded to, both structure-based drug design (SBDD) and QSAR have previously been hyped as coming to the rescue of medicinal chemists; can we learn anything from the development arc of these methods to guide our way forward? A popular view is generally "overhyped but then have found their place" It is also interesting to note the well-documented "AI effect" which can be summarized as: "that as soon as a problem is solved it's rebranded and "not AI" but "merely computation" or a "brute force approach". ${ }^{25}$ There is an obvious parallel in 
both QSAR and SBDD, in that once methods become successful they are rebranded possibly to escape from the initial hype that supported their early development. An early example of this is Hansch's seminal work in QSAR, looking at sigma and pi parameters to choose substituents. ${ }^{26}$ Here many chemists were very cynical and resistant to the use of simple QSAR, however once reformulated by John Topliss, ${ }^{27}$ the Topliss tree has become a standard method for looking at substituents and considering what might be driving activity, all without a chemist having to consider linear regression (the machine learning method of the 1960's). For an excellent summary of the of the development of QSAR see the 2014 perspective by Tropsha et al. ${ }^{28}$ QSAR has found a place in certain key domains: where there are large datasets such as in computational toxicology particularly in the evaluation of risk for environmental toxins ${ }^{29}$ and flagging potential carcinogens and mutagens. ${ }^{30}$ In these cases computational methods are so well accepted as to be a regulatory requirement. Martin reviewing what methods have worked at Abbot in 2006, highlights the use of expert systems and particularly the encoding of sub-structural flags as warnings for chemists. ${ }^{31}$ This concept has become even more widely adopted with the publication of academic equivalents of corporate warning flags in the PAINS filters, ${ }^{32}$ where the principle is now well-adopted though the detail of implementation remains controversial. ${ }^{33}$ This is a mark of the maturing of the area, where more detailed publication and discussion of the methods shows that adoption is in serious progress. Cumming, Davis and co-workings at AstraZeneca similarly show the impact substructural flags have made ${ }^{34}$ but also draw attention to the use of QSAR models in predicting solubility and ADMET properties. Examples of impact include "AstraZeneca's global HERG QSAR model has also contributed to the reduction in the synthesis of 'red flag' compounds (compounds that are measured to have an HERG potency of $<1 \mu \mathrm{M}$ ), from $25.8 \%$ of all compounds tested in 2003 to only $6 \%$ in 2010.” Here there were several distinguishing features: firstly, a 
psychological effect whereas automatically built QSAR models built from very large datasets were introduced and the medicinal and computational chemists needed to trust that the automated models were as good as or better than bespoke built models; secondly the positive effect that management behaviors can have on the adoption of predictive methods. The role of managers expecting predictions to be done before synthesis and then engaging in a dialogue when the results arrive can clearly have a significant effect.

The other large area QSAR has made an impact on is in in silico ADMET profiling. Here there is an interesting cross industry view ${ }^{35}$ (from nine companies) that, beyond the technical implementations, "Models can only be truly impactful if the organization embraces and fosters an in silico culture; this requires a diversified approach involving in silico scientists, DMPK scientists (in vitro and in vivo), chemists and, to some extent, management." The lower impact of QSAR in other aspects of drug hunting has been assigned to a lack of feedback of measured data to the challenge and improve the algorithms and descriptors in use, and even worse: "QSAR disappoints because we have largely exchanged the tools of the scientific method in favor of a statistical sledgehammer", a prescient warning for those applying newer machine learning methods. ${ }^{36}$ An excellent guide for those aspiring to do better QSAR modelling has been provided by Dearden and collegues $^{37}$ which should be compulsory reading for those developing all machine learning systems dealing with biological data. A critical common feature where QSAR has been more successful has been where there have been large data sets. However even in small data sets modelling may summarize the data in an unbiased way, and where the models are sufficiently explainable, may direct a team as to where more data needs to be gathered or where there are early indications of SAR. 
A retrospective analysis from Genentech ${ }^{38}$ highlights several key strategies important for the successful implementation of both QSAR and structure based drug design. A significant part of the analysis is the way in which computational chemists and medicinal chemistry are organized to work together. Computational chemists are not over stretched, with a maximum of 2 projects to deliver against, which enables them to be full project team members, not just "service providers". This aligns their performance management with that of the project teams which clearly incentivizes computational and medicinal chemists to work together. Considerable effort is described in delivering software integration so that medicinal chemists can easily perform routine tasks and computational chemists are freed to conduct specialized analysis and modelling. Software development is focused on integration and automation of informatics tasks to ensure that the data is available to work on and effort is concentrated on the critical task of project teams: deciding on which experiment to do next.

A more descriptive culture is discussed by Stahl and co-workers from Roche in Basle in their publications on knowledge capture in wiki format, ${ }^{39}$ a set of case studies of what actually has worked in their experience ${ }^{40}$ and a reflection on the process of attempting to capture the research process itself. ${ }^{41}$ Here again, the challenges of the interaction between the medicinal chemist and computational chemists and informaticians is emphasized, in particular in the need for the software engineers to understand the actual problems that the medicinal chemists are facing. In particular there is an appreciation of the richness and complexity of the research process. There is the concern that in capturing the process in software some critical aspects of understanding may be lost: "Earlier generations of medicinal chemists worked with pen and paper to jot down ideas as well as to document their work. Are today's more rigid electronic systems a hindrance to creativity?". The ideal would be to have an electronic lab journals that would automatically 
classify and cross link concepts during the ideation process of medicinal chemistry. Some understanding of what may be possible is shown in the automated natural language processing of large patent corpuses where chemical names can be linked to structures, reference numbers correctly identified and tables extracted. ${ }^{42}$ This would enable a comparison to be made between a more 'free wheeling' approach to creative medicinal chemistry and one where work flows are more enforced in order to work easily with computational tools.

At the opposite end of the spectrum is the recent disclosure from the Molecular Design Group at GSK where they introduce their approach to creating a fully integrated automated molecular design system developed internally named BRADSHAW. ${ }^{43}$ A set of common computational medicinal chemistry tasks have been automated, and exposed via a web interface. These were chosen to be those both frequently needed and where a lower level of specialist knowledge is required to carry them out. The critical tasks were identified as molecule generation, compound profiling, multiparameter ranking, substructure filtering, active learning, experimental design for array reagent selection and automated Free-Wilson analysis. Currently it is focused on cheminformatics and machine learning methods recognizing that 3D and physics-based techniques such as FEP+ are still in a development phase where more expert control is needed. Having built the system there is however a brief discussion on how it fits into the working of a chemistry lead optimization team : "Is it as a complement to the creativity of the team, or should the automated system become the fundamental workhorse for the team which is complemented by suggestions and decisions by the human supervisors? We feel these answers will become clearer as the platform is further tested in prospective applied scenarios". This challenge is at the heart of how well the emerging generation of machine learning and AI methods will change our disciplines. 


\section{Taking control of AI in medicinal chemistry}

With the experience we have already gained as a field we should be able to consider how the new generation of machine learning tools will impact, and even more importantly how beyond machine learning, how AI methods can contribute. Taking the algorithms of image ${ }^{44}$ and text analysis ${ }^{45}$ and applying them to ligand interactions,${ }^{46}$ quantum mechanics ${ }^{47}$ and QSAR; ${ }^{48}$ the new dogma is that data-driven representations can displace the physical chemistry and quantum mechanical understanding of molecules, and that synthetic chemistry knowledge can be replaced by data-mining based reaction and route prediction. ${ }^{49-51}$ The hope (and marketing) is that all that expensive expertise in both medicinal and computational chemistry can be at least equaled if not improved upon by data-driven methods. As many have pointed out however, in misquoting Peter Norvig of Google "data is unreasonably effective" 52 - you do need an eye watering amount of it before machine learning methods can deliver significant improvements. This is where the challenge comes: the new generation of machine learning methods are indeed highly effective with enough data but, unlike in many of the success stories where data has been harvested from consumers(in the case of commercial recommender systems and satellite navigation, at low to zero cost) the cost of making and testing compounds still is in the $\$ 100$ 's to $\$ 1000$ s per compound. It may be that the past poor behaviors of some medicinal chemists now come home to haunt them. Although reaction prediction software is currently expensive, training on easily available and highly relevant patent chemistry enables computational chemists to access synthetic feasibility and route planning estimates more easily than persuading a recalcitrant medicinal chemist. ${ }^{51}$ Coupling this with access to CROs who will synthesize compounds on demand, the opportunity for computational chemists to bypass medicinal chemists should be appealing. There is, of course, a 
catch. Currently the models created by machine learning systems are clearly well below what would be needed for them to be reliable..$^{53,54}$ Those with little experience in real drug hunting having read the literature could hold the view that it should be relatively simple to automate. Just follow the Rule-of-Five, reduce lipophilicity, make common substitutions and cyclize / de-cyclize and all will be well. Unfortunately, the medicinal chemistry literature often paints too rosy a view of how a team rationally followed a logical track with the occasional strike of good fortune (the unexpectedly good result beloved of patent attorneys). It is easy to see how inexperienced novice drug hunters applying undertrained AI systems could tip our disciplines into the AI hype cycle "storm of disillusionment". In the management literature this is well recognized as an issue for highly skilled isolated professionals as the "zoom into doom loop" 55 where rather than evaluating where systems are going wrong and improving them, the defensive emotional response is to disparaged systems (or their creators) and abandon them. In this situation our goal should be to navigate a better path rather than blame each other.

\section{A way forward}

So what to do? Several organizations have proposed the "Augmented or enhanced chemist" option - the "Centaur system" with the machine learning and AI "horses body" doing the heavy lifting of calculations and data wrangling, with the human head making the strategic decisions and keeping the system from making ridiculous suggestions or not backing off quickly enough when an inaccurate measurement leads you astray. ${ }^{56}$ This is an appealing image and has good precedent in chess where centaur teams of multiple AI systems with humans can outplay any single grandmaster. ${ }^{57}$ However, drug hunting is not a game, and the risk is that in the same way that 
offshoring synthetic chemistry in the 1990's was marketed as "enhancing teams with additional flexible resource" enhancement may rapidly turn to replacement once the extra resource is good enough. It also does not address the critical issue that current systems built on small data sets are still not, by objective measures, very good.

In the short term the need to deliver compounds to the clinic will mean that the human part of a centaur team is essential. Although there has been significant work in quantifying the applicability domain of models, these are essentially models of the perimeter of a system and validating the domain of applicability appears to be as hard as validating the model itself. ${ }^{58-62}$ Once again, the developments in other fields with vastly more data do not easily transfer to drug hunting with smaller data sets and hard to describe molecules. There is still a significant role for chemists to play in assessing whether a prediction should be in or out of domain of a model. At a technical level, the number of possible compounds that conservatively change a hit/lead compound is still staggeringly high. This means strategic decision making and situational analysis: understanding what phase a project is in, what the critical issues are and what data and techniques to apply to narrow the prospective chemistry space will be a human task for a while, but we should still heed the words of John von Neumann(vide infra), if we can gather the data and choose to attempt encoding it, even these tasks may be automatable in time. One can imagine using the established Biopharmaceutical Classification System $^{63}$ as one method for assigning the situation a lead optimization project is in and defining the strategy to apply. The automation of strategic analysis been proved possible complex "toy" systems,${ }^{64}$ to assume it requires uniquely human skills is probably displaying a certain hubris. 
For the next decade a more secure approach is to develop explainable and auditable AI systems and rigorously measure and improve their quality. While systems are still unreliable, we need to understand their working and direct compound synthesis and data gathering to where knowledge is missing to improve them. This should be the shared goal of medicinal and computational chemists, to generate the data and build the models that can go forward to deliver better drug hunting systems. Medicinal chemists cannot simultaneously claim that medicinal chemistry is some kind of mystical art that only they have the insights to understand and, at the same time, maintain you can train a medicinal chemist to be competent in 5 years after a $\mathrm{PhD}$ in synthetic chemistry. To quote John von Neumann "You insist that there is something a machine cannot do. If you tell me precisely what it is a machine cannot do, then I can always make a machine which will do just that." Within this framework, the computational chemistry community also needs to be a part of generating new compounds and data, and changing their systems accordingly. Despite the discomfort, those building models need to appreciate, possibly by personal experience the challenge experimentalists face. It is slowly becoming more common for a medicinal chemistry lecture to include some computational chemistry as a rationale for why a particular course was taken in the development of a series. In the same way, we need to see more computational chemistry presentations include "so we went and had the following compounds made, tested and then refactored our model when the results didn't match our prediction". Even: "I joined the team that made the compounds and saw the effect low solubility had on measurements." Both of these obviously represent a threat to established practice, and it will be a challenge for us as a discipline to step away from some of our more defensive reflexes including the pride in ignorance of computational tools, or “it wouldn't be safe for me to be back in a lab". As described earlier, both medicinal and computational chemists have a strong sense of professional identity, and both of 
these pictures of change are difficult because they represent a change in our own perceived identity. For competent, confident and curious scientists this should not present a problem as key parts of their professional skill set are pragmatic self-appraisal and learning new skills; however, we need to recognize that the for the less confident individuals more support may be needed. There is precedence in many other domains in adopting a more "productive reasoning" strategy, generating testable data, building monitoring into AI systems to continuously report their performance and openly discussing the professional threats AI systems bring. ${ }^{21}$

This issue of the Journal of Medicinal Chemistry is a start point in addressing the opportunities for the application of artificial intelligence to medicinal chemistry. However there is much more to do: we need to set a new set of editorial and reviewer expectations that modelling publications should be expected to include new compounds made and tested and, equally, medicinal chemistry publications should always show how the compounds proposed performed compared to models, or if they were inspired by data analysis how that that process was applied. This will drive collaborative working and improve the training of $\mathrm{PhD}$ students not to be masters of multiple domains, but to be able to collaborate better with other specialists. Another lever to apply is to funding and reviewing bodies. We need to set the expectation that for research in medicinal chemistry and the drug hunting aspects of computational chemistry the combination of calculation, making and testing compounds is a minimum. Whether this is public, corporate or charitable support, all should hold to a better standard. Inevitably this will drive academic training to expect the combination of calculation and measurement, and to have the next generation of researchers equipped to move our disciplines forward. It may result in a fundamental shift in the behaviors of chemists; if working in cross disciplinary teams becomes the norm in our training, we may become better team workers as a discipline. Though uncomfortable, this is not unheard of in other physical 
sciences where 'big physics' requires large interdisciplinary teams to work together on long term complex projects. We have just been funded to work more in academic silos than perhaps has been good for us. A further marker for the current state of play is the organization of scientific meetings and conferences. Currently, medicinal and computational chemistry meetings operate in isolated parallel streams. American Chemical Society National meetings are one of the most obvious examples of this where the MEDI and CINF/COMP sections operate in ignorance of each other despite the incredible value both communities could gain from understanding each other's problems and ideas better. Usually they are even geographically separated, making it hard for the casually curious to swap between streams. Similarly, the Gordon Research Conferences for Computer Aided Drug Design (CADD) and Medicinal Chemistry operate as two distinct groups with only a rare individual crossing between the two. The opportunity for learned societies to enable cross disciplinary meetings is obvious. We need to create spaces where computational and medicinal chemists can meet and share their challenges and successes to drive forward drug hunting. A challenge here is that conferences represent communities of practice and the organizing groups of regular conferences enjoy some positional power. Redrawing the boundaries of these communities is a challenge to those currently in power: the risk is that in not evolving they may become irrelevant. As ever, here is little new under the sun and Paul Janssen is quoted as having recognized the general version of the problem "A good scientist is someone who succeeds in getting the different scientific disciplines to work in harmony with one another" ${ }^{65}$ This is, of course, a choice, the alternative is to allow the tension between medicinal and computational chemists to hobble the development of AI systems, which then fail to deliver improvements and fulfill the easy predictions of the naysayers and medicinal chemistry suffers another AI winter. In 
those circumstances though both disciplines will have played a defensive strategy and won some small scale security in the short term: in the longer term we will all have all lost.

It will not be enough however for just computational and medicinal chemists to collaborate to create the AI systems that will advance compounds to the clinic faster. Chemists work within organizations and therefore there is a role for their managers to take on. Making the changes to how chemists work will require addressing two sets of challenges for research managers. Firstly, they have a critical role in demonstrating that the adoption of AI methods is to become part of the new drug hunting culture. Scientists are adept at observing their managers and distinguishing words from actions and noting which is to be followed. There are two classes of actions: "superficial" actions such as statements of organizational philosophy, physical spaces, organizational rites like internal prizes, and organizational structures, and the "core" change activities such as who is rewarded, promoted and removed from organizations, which departments are resourced especially in constrained times and how leaders respond to crises. Ideally the superficial and core activities would be aligned, but if the superficial is changed while the core remains the same, or even undermines the declared intent, wise chemists will pay attention and avoid following fashion where clearly managers are not "walking the talk". These are wellestablished organizational principles. ${ }^{66}$ The second aspect is for chemistry managers themselves to recognize and respond to the challenge that AI brings in triggering a defensive response in themselves and their teams. This in itself is likely to be problematic. Most chemistry managers have the experience of coming from either the medicinal or computational side of chemistry and thus will be less familiar with the other discipline. They will by virtue of seniority in many cases have lived through previous hype cycles of QSAR, automated retrosynthetic analysis, structurebased drug design, high throughput screening, combinatorial chemistry and automated chemistry. 
It would be easy to see the current wave of AI methods as being another wave destined to shine for a brief while then fall back into finding its place. A cautious manager would allow some experimentation and watch the external environment while not committing to real change while allowing others to take the risks, while delivering current projects into the clinic. It's easy to sell this approach as being safe, showing strong leadership, rational and of course keeping focused on the essential immediate task of delivering new therapies for patients. We believe, however, that significantly more courage is needed, but that leaders do not need to buy the hype uncritically. Possibly uniquely, one of the facets of AI is the need to gather data for the application of machine learning methods. So rather than just accepting the promises, systems must be put in place that gather real information on whether and how well systems work and use this data to evaluate and improve them. In turn, AI systems can then be constantly monitored to look for errors and drift and seek opportunities to feed back to computational chemists the areas for improvement in the science. This means that the cautious manager can extract some early benefits from a system, and where they need to justify further investment in automation with results, be able to show how the system is performing on a smaller scale before larger investment. Real embedding of AI methods in chemistry will require the automation of compound design, synthesis, testing and then optimization. Automated data collection will enable the application of machine learning methods to all aspects of the chemical part of drug discovery, and then analysis, further validation and optimization. Some companies have already started to capture how hypotheses deliver when compounds are made and tested, including the proposals from automated systems into this seems an obvious extension. ${ }^{39,41,67}$ There is evidence that when this is done and managers are more assertive, chemists will respond accordingly as AstraZeneca Sodertalje demonstrated in using QSAR models for solubility to improve the compounds synthesized. ${ }^{34}$ An implementation 
challenge here is that the monitoring aspect of AI implementation is more software and systems engineering than science. The learning from the implementation of high throughput screening, and fragment-based drug design is that to really gain the benefits, processes need to be industrialized which means that $\mathrm{AI}$ systems need to be moved into a production engineering framework, with testing, monitoring and planned maintenance. A challenge for the management of such groups is that the people that are interested in creating new systems are not the people to industrialize them. In a research environment, the people who industrialize systems need to be properly recognized for the contribution they make. Production systems should be judged on scalability, reliability and robustness rather than novelty and performance which are more the goals for research scientists. The demands put in place to make a production system reliable makes them less flexible which may be frustrating for the computational chemist, however to deliver working systems for project drug hunters, industrialization is essential.

Once systems are reliably in place, there is the opportunity to move into active learning: the systematic approach to generating experiments where some fraction of the compounds or reaction conditions suggested are proposed as being the "best new compounds / reactions" and another fraction are proposed deliberately to enhance understanding. ${ }^{68}$ This is usually described as the exploit/explore balance, a completely familiar concept to medicinal chemists who talk about "exploring SAR". This also shifts the dialogue between the three parties - with data gathered the value of AI approaches can be assessed, medicinal chemists can be challenged on their application of methods, and computational chemists on the accuracy and applicability domains of approaches adopted. Managers themselves have evidence to work from in justifying investment in equipment, training and the change process itself. 


\section{Are the behaviors we have observed culture specific?}

This article is written from the perspective of the large American-European Pharmaceutical company sector. Over the last seventy years the organizations, systems and behaviors we have evolved in this culture have brought us this far, and delivered some superb therapies. New entrants to drug discovery from either the technology sector or other geographical regions have the option to choose new behaviors. Those coming from a pure data science background may hold the belief that "no domain knowledge is necessary" and models can be inferred with sufficient data. It can be a rude awakening to discover that there isn't enough data (by orders of magnitude) and that both biological and chemical systems can be remarkably unforgiving and complex. Finding the right way to incorporate the background domain knowledge of established drug hunting while exploiting the innovations of modern AI approaches is a significant challenge. There is the risk that with the need to acquire staff with critical domain skills in medicinal and computational chemistries they may also import the same defensive behavioral models that are the root of many of our issues. Alternatively, they may be able to start with a cleaner slate, or perhaps greater awareness of the pitfalls ahead and find it easier to overturn unhelpful inherited customs and practices.

\section{First steps in the way forward}

We believe that in developing high quality artificial intelligence systems to aid us in drug hunting the collaboration between medicinal and computational chemists is essential. A different way of working, embedding data capture from the start, for every aspect of drug design, including 
hypothesis generation by human or machine, will allow us to make comparisons and improvements based on evidence rather than falling back into defensive behaviors. Sometimes, it can be easy to lose sight of why most of us are involved in medicinal and computational chemistry: the science is in the service of clinical improvement. Although the changes that the integration of AI systems brings will require a challenge to our sense of what it is to be a medicinal or computational chemist, the satisfaction of delivering therapies to the clinic makes the price worthwhile. We need AI to help us because the low hanging fruit are long gone: we need to apply our very best approaches to deliver therapies for new generations.

\section{Corresponding Author}

* Ed J Griffen, MedChemica Ltd, Alderley Park, Macclesfield, Cheshire. SK10 4TG.

\section{Author Contributions}

The manuscript was written through contributions of all authors. All authors have given approval to the final version of the manuscript.

\section{Biographies}

Ed. J. Griffen obtained his Ph.D. from Imperial College, London, and undertook postdoctoral research at the University of Waterloo-Kitchener, Canada. He joined Zeneca Pharmaceuticals in medicinal chemistry working in the CNS, infection, oncology, and chemical biology areas. Taking a secondment into the computational chemistry group, he co-developed matched molecular pair tools to quantify medicinal chemistry approaches. In 2012 he cofounded MedChemica Ltd. He has taught medicinal chemistry courses at the University of Manchester and in Mumbai and 
AstraZeneca courses in the U.K., France, and India. He has contributed to four compounds that have entered clinical development, is a named inventor on 16 patents and co-authored 18 articles, book chapters, and a textbook. His interests are in improving data driven medicinal chemistry practice.

Alexander G. Dossetter gained his Ph.D. from Nottingham University and after postdoctoral research at Harvard University joined AstraZeneca. He has 13 years of experience in medicinal chemistry spread across oncology (hormonal and kinase inhibitors), inflammation (OA and RA, enzyme inhibitors, and GPCR targets), and diabetes (obesity, GPCR, and enzyme inhibitors). In 2012, he cofounded MedChemica Limited to use matched molecular pair analysis to accelerate medicinal chemistry. Al is an enthusiastic advocate for science and is frequently invited to present his research.

Andrew G. Leach obtained his Ph.D. from the University of Cambridge and undertook postdoctoral research at UCLA, supported by the Fulbright scheme. He returned to the UK to join AstraZeneca as a computational chemist and worked in oncology, diabetes, and obesity. In 2012, he became one of the cofounders of MedChemica Limited and also joined Liverpool John Moores University as a lecturer. He is currently a Lecturer in Pharmaceutical Chemistry in the School of Pharmacy at the University of Manchester. His interests include all aspects of computation to support drug discovery and medicinal chemistry as well as the use of quantum mechanics to unravel interesting reaction mechanisms. He has published more the 70 articles, 5 book chapters and is a named inventor on 12 patents. 


\section{ORCID}

Ed J. Griffen

Alexander G. Dossetter

Andrew G. Leach
0000-0003-0859-554X

0000-0002-4181-3193

0000-0003-1325-8273

\section{ACKNOWLEDGMENTS}

The authors thank Neil Raval of CCMR Consulting Ltd for very useful conversations in the field of organizational structures and change management.

\section{ABBREVIATIONS}

AI, artificial intelligence; CRO, contract research organization; PAINS Pan Assay Interference Compounds; FEP Free Energy Perturbation.

\section{REFERENCES}

(1) Feigenbaum, E. A. Some Challenges and Grand Challenges for Computational Intelligence. Journal of the ACM 2003, 50 (1), 32-40. https://doi.org/10.1145/602382.602400.

(2) Turing, A. M. I. - Computing Machinery And Intelligence. Mind 1950, LIX (236), 433-460. https://doi.org/10.1093/mind/LIX.236.433.

(3) The Cambridge Handbook of Expertise and Expert Performance; Ericsson, K. A., Ed.; Cambridge University Press: Cambridge ; New York, 2006.

(4) Rosenblatt, F. The Perceptron: A Probabilistic Model for Information Storage and Organization in the Brain. Psychological Review 1958, 65 (6), 386-408. https://doi.org/10.1037/h0042519. 
(5) Pharma R\&D Returns Fall To Lowest Level In Nine Years https://www2.deloitte.com/uk/en/pages/press-releases/articles/pharma-r-and-d-returns-fallto-lowest-level-in-nine-years.html (accessed Nov 28, 2019).

(6) Scannell, J. W.; Bosley, J. When Quality Beats Quantity: Decision Theory, Drug Discovery, and the Reproducibility Crisis. PLOS ONE 2016, 11 (2), e0147215. https://doi.org/10.1371/journal.pone.0147215.

(7) Jordan, A. M. Artificial Intelligence in Drug Design-The Storm Before the Calm? ACS Medicinal Chemistry Letters 2018, $9 \quad$ (12), 1150-1152. https://doi.org/10.1021/acsmedchemlett.8b00500.

(8) Hawkins, P. Virtual Screening At Ultra-Large Scale: 1.5 Billion And Counting - Webinars http://www.healthtech.com/openeye-scientific-virtual-screening-at-ultra-large-scale/ (accessed Nov 28, 2019).

(9) Hawkins, P. Webinar: REAL-ly Large-Scale Virtual Screening - Traversing Enormous Regions of Chemical Space with the GPU and CPU. https://www.eyesopen.com/news/webinar-2019-really-large-virtual-screening (accessed Nov 28, 2019).

(10) About Torx ${ }^{\mathrm{TM}}$ https://www.torx-platform.com/about-torx/ (accessed Nov 28, 2019).

(11) MedChemica Ltd. MCPairs https://www.medchemica.com/products/ (accessed Nov 28, 2019).

(12) LiveDesign® | Schrödinger https://www.schrodinger.com/livedesign/ (accessed Nov 28, 2019). 
(13) Molecular Operating Environment (MOE) । MOEsaic I PSILO https://www.chemcomp.com/Products.htm\#MOEsaic-SAR_Explorer (accessed Nov 28, 2019).

(14) Ritchie, T. J.; McLay, I. M. Should Medicinal Chemists Do Molecular Modelling? Drug Discovery Today 2012, 17 (11-12), 534-537. https://doi.org/10.1016/j.drudis.2012.01.005.

(15) Bodkin, M. J. Why Don't We See a Greater Uptake of Computational Chemistry Approaches by the Medicinal Chemistry Community? Future Medicinal Chemistry 2012, 4 (15), 1889-1891. https://doi.org/10.4155/fmc.12.154.

(16) Brown, S. P.; Muchmore, S. W.; Hajduk, P. J. Healthy Skepticism: Assessing Realistic Model Performance. Drug Discovery Today 2009, 14 (7-8), 420-427. https://doi.org/10.1016/j.drudis.2009.01.012.

(17) Nicholls, A. Confidence Limits, Error Bars and Method Comparison in Molecular Modeling. Part 2: Comparing Methods. J Comput Aided Mol Des 2016, 30 (2), 103-126. https://doi.org/10.1007/s10822-016-9904-5.

(18) Carlson, H. A. Check Your Confidence: Size Really Does Matter. J. Chem. Inf. Model. 2013, 53 (8), 1837-1841. https://doi.org/10.1021/ci4004249.

(19) Wenlock, M. C.; Carlsson, L. A. How Experimental Errors Influence Drug Metabolism and Pharmacokinetic QSAR/QSPR Models. J. Chem. Inf. Model. 2015, 55 (1), 125-134. https://doi.org/10.1021/ci500535s.

(20) Kutchukian, P. S.; Vasilyeva, N. Y.; Xu, J.; Lindvall, M. K.; Dillon, M. P.; Glick, M.; Coley, J. D.; Brooijmans, N. Inside the Mind of a Medicinal Chemist: The Role of Human Bias in Compound Prioritization during Drug Discovery. PLoS ONE 2012, 7 (11), e48476. https://doi.org/10.1371/journal.pone.0048476. 
(21) Argyris, C. Organizational Traps: Leadership, Culture, Organizational Design; Oxford University Press: Oxford ; New York, 2010.

(22) Argyris, C. Overcoming Organizational Defenses: Facilitating Organizational Learning; Allyn and Bacon: Boston, 1990.

(23) Moltke, H. G. von. Moltke on the Art of War: Selected Writings; Presidion Press: New York, 1996.

(24) Brown, D. G.; Boström, J. Where Do Recent Small Molecule Clinical Development Candidates Come From? J. Med. Chem. 2018, 61 (21), 9442-9468. https://doi.org/10.1021/acs.jmedchem.8b00675.

(25) Griffen, E. J.; Dossetter, A. G.; Leach, A. G.; Montague, S. Artificial Intelligence in Medicinal Chemistry. In Burgers Medicinal Chemistry, Drug Discovery and Development; Abraham, D. J., Ed.; John Wiley \& Sons, Inc., 2020.

(26) Hansch, Corwin.; Fujita, Toshio. P- $\sigma-\pi$ Analysis. A Method for the Correlation of Biological Activity and Chemical Structure. J. Am. Chem. Soc. 1964, 86 (8), 1616-1626. https://doi.org/10.1021/ja01062a035.

(27) Topliss, J. G. Utilization of Operational Schemes for Analog Synthesis in Drug Design. J. Med.Chem. 1972, 15 (10), 1006-1011. https://doi.org/10.1021/jm00280a002.

(28) Cherkasov, A.; Muratov, E. N.; Fourches, D.; Varnek, A.; Baskin, I. I.; Cronin, M.; Dearden, J.; Gramatica, P.; Martin, Y. C.; Todeschini, R.; Consonni, V.; Kuz'min, V. E.; Cramer, R.; Benigni, R.; Yang, C.; Rathman, J.; Terfloth, L.; Gasteiger, J.; Richard, A.; Tropsha, A. QSAR Modeling: Where Have You Been? Where Are You Going To? J. Med.Chem. 2014, 57 (12), 4977-5010. https://doi.org/10.1021/jm4004285. 
(29) European Chemicals Agency. Illustrative Example with the OECD QSAR Toolbox Workflow. Part 1, Part 1,; ECHA: Helsinki, 2014.

(30) Teasdale, A. ICH M7: Assessment and Control of DNA Reactive (Mutagenic) Impurities in Pharmaceuticals to Limit Potential Carcinogenic Risk. In ICH Quality Guidelines; Teasdale, A., Elder, D., Nims, R. W., Eds.; John Wiley \& Sons, Inc.: Hoboken, NJ, USA, 2017; pp 667-699. https://doi.org/10.1002/9781118971147.ch24.

(31) Martin, Y. C. What Works and What Does Not: Lessons From Experience in a Pharmaceutical Company. QSAR \& Combinatorial Science 2006, 25 (12), 1192-1200. https://doi.org/10.1002/qsar.200610102.

(32) Baell, J. B.; Holloway, G. A. New Substructure Filters for Removal of Pan Assay Interference Compounds (PAINS) from Screening Libraries and for Their Exclusion in Bioassays. J. Med. Chem. 2010, 53 (7), 2719-2740. https://doi.org/10.1021/jm901137j.

(33) Capuzzi, S. J.; Muratov, E. N.; Tropsha, A. Phantom PAINS: Problems with the Utility of Alerts for P an- A Ssay IN Terference Compound S. J. Chem. Inf. Model. 2017, 57 (3), 417427. https://doi.org/10.1021/acs.jcim.6b00465.

(34) Cumming, J. G.; Davis, A. M.; Muresan, S.; Haeberlein, M.; Chen, H. Chemical Predictive Modelling to Improve Compound Quality. Nat Rev Drug Discov 2013, 12 (12), 948-962. https://doi.org/10.1038/nrd4128.

(35) Lombardo, F.; Desai, P. V.; Arimoto, R.; Desino, K. E.; Fischer, H.; Keefer, C. E.; Petersson, C.; Winiwarter, S.; Broccatelli, F. In Silico Absorption, Distribution, Metabolism, Excretion, and Pharmacokinetics (ADME-PK): Utility and Best Practices. An Industry Perspective from the International Consortium for Innovation through Quality in 
Pharmaceutical Development: Miniperspective. Journal of Medicinal Chemistry 2017, 60 (22), 9097-9113. https://doi.org/10.1021/acs.jmedchem.7b00487.

(36) Johnson, S. R. The Trouble with QSAR (or How I Learned To Stop Worrying and Embrace Fallacy). J. Chem. Inf. Model. 2008, 48 (1), 25-26. https://doi.org/10.1021/ci700332k.

(37) Dearden, J. C.; Cronin, M. T. D.; Kaiser, K. L. E. How Not to Develop a Quantitative Structure-Activity or Structure-Property Relationship (QSAR/QSPR). SAR and QSAR in Environmental Research 2009, $20 \quad$ (3-4), 241-266. https://doi.org/10.1080/10629360902949567.

(38) Tsui, V.; Ortwine, D. F.; Blaney, J. M. Enabling Drug Discovery Project Decisions with Integrated Computational Chemistry and Informatics. J Comput Aided Mol Des 2017, 31 (3), 287-291. https://doi.org/10.1007/s10822-016-9988-y.

(39) Mayweg, A.; Hofer, U.; Schnider, P.; Agnetti, F.; Galley, G.; Mattei, P.; Lucas, M.; Boehm, H.-J. ROCK: The Roche Medicinal Chemistry Knowledge Application? Design, Use and Impact. Drug Discovery Today 2011, $16 \quad$ (15-16), 691-696. https://doi.org/10.1016/j.drudis.2011.03.005.

(40) Kuhn, B.; Guba, W.; Hert, J.; Banner, D.; Bissantz, C.; Ceccarelli, S.; Haap, W.; Körner, M.; Kuglstatter, A.; Lerner, C.; Mattei, P.; Neidhart, W.; Pinard, E.; Rudolph, M. G.; SchulzGasch, T.; Woltering, T.; Stahl, M. A Real-World Perspective on Molecular Design: Miniperspective. J. Med. Chem. 2016, $59 \quad$ (9), 4087-4102. https://doi.org/10.1021/acs.jmedchem.5b01875.

(41) Stahl, M.; Baier, S. How Many Molecules Does It Take to Tell a Story? Case Studies, Language, and an Epistemic View of Medicinal Chemistry. ChemMedChem 2015, 10 (6), 949-956. https://doi.org/10.1002/cmdc.201500091. 
(42) Lowe, D.; Senger, S.; Sayle, R. A. Automatic Extraction of Bioactivity Data from Patents, https://www.slideshare.net/NextMoveSoftware/automatic-extraction-of-bioactivity-datafrom-patents-74402139, (accessed May 4, 2020).

(43) Green, D. V. S.; Pickett, S.; Luscombe, C.; Senger, S.; Marcus, D.; Meslamani, J.; Brett, D.; Powell, A.; Masson, J. BRADSHAW: A System for Automated Molecular Design. $J$ Comput Aided Mol Des 2019. https://doi.org/10.1007/s10822-019-00234-8.

(44) Goh, G. B.; Siegel, C.; Vishnu, A.; Hodas, N. O.; Baker, N. Chemception: A Deep Neural Network with Minimal Chemistry Knowledge Matches the Performance of ExpertDeveloped QSAR/QSPR Models. arXiv:1706.06689 [cs, stat] 2017.

(45) Bjerrum, E. J. SMILES Enumeration as Data Augmentation for Neural Network Modeling of Molecules. arXiv:1703.07076 [cs] 2017.

(46) Feinberg, E. N.; Sur, D.; Wu, Z.; Husic, B. E.; Mai, H.; Li, Y.; Sun, S.; Yang, J.; Ramsundar, B.; Pande, V. S. PotentialNet for Molecular Property Prediction. ACS Central Science 2018, 4 (11), 1520-1530. https://doi.org/10.1021/acscentsci.8b00507.

(47) Smith, J. S.; Isayev, O.; Roitberg, A. E. ANI-1: An Extensible Neural Network Potential with DFT Accuracy at Force Field Computational Cost. Chem. Sci. 2017, 8 (4), 3192-3203. https://doi.org/10.1039/C6SC05720A.

(48) Chen, H.; Engkvist, O.; Wang, Y.; Olivecrona, M.; Blaschke, T. The Rise of Deep Learning in Drug Discovery. Drug Discovery Today 2018, 23 (6), 1241-1250. https://doi.org/10.1016/j.drudis.2018.01.039.

(49) Badowski, T.; Gajewska, E. P.; Molga, K.; Grzybowski, B. A. Synergy Between Expert and Machine-Learning Approaches Allows for Improved Retrosynthetic Planning. Angew. Chem. Int. Ed. 2019. https://doi.org/10.1002/anie.201912083. 
(50) Klucznik, T.; Mikulak-Klucznik, B.; McCormack, M. P.; Lima, H.; Szymkuć, S.; Bhowmick, M.; Molga, K.; Zhou, Y.; Rickershauser, L.; Gajewska, E. P.; Toutchkine, A.; Dittwald, P.; Startek, M. P.; Kirkovits, G. J.; Roszak, R.; Adamski, A.; Sieredzińska, B.; Mrksich, M.; Trice, S. L. J.; Grzybowski, B. A. Efficient Syntheses of Diverse, Medicinally Relevant Targets Planned by Computer and Executed in the Laboratory. Chem 2018, 4 (3), 522-532. https://doi.org/10.1016/j.chempr.2018.02.002.

(51) Segler, M. H. S.; Preuss, M.; Waller, M. P. Planning Chemical Syntheses with Deep Neural Networks and Symbolic AI. Nature 2018, 555 (7698), 604-610. https://doi.org/10.1038/nature25978.

(52) Halevy, A.; Norvig, P.; Pereira, F. The Unreasonable Effectiveness of Data. IEEE Intelligent Systems 2009, 24 (2), 8-12. https://doi.org/10.1109/MIS.2009.36.

(53) Ma, J.; Sheridan, R. P.; Liaw, A.; Dahl, G. E.; Svetnik, V. Deep Neural Nets as a Method for Quantitative Structure-Activity Relationships. J. Chem. Inf. Model. 2015, 55 (2), 263274. https://doi.org/10.1021/ci500747n.

(54) Maggiora, G. M. On Outliers and Activity Cliffs - Why QSAR Often Disappoints. J. Chem. Inf. Model. 2006, 46, 1535. https://doi.org/10.1021/ci060117s.

(55) Argyris, C. Teaching Smart People How to Learn. Harvard Business Review. May 1, 1991.

(56) Centaur Chemist ${ }^{\mathrm{TM}}$ I AI Drug Discovery I Exscientia https://www.exscientia.com (accessed Nov 28, 2019).

(57) Cassidy, M.; Signifyd, C. storyteller at; News, formerly a columnist at the S. J. M. Centaur Chess Shows Power of Teaming Human and Machine https://www.huffpost.com/entry/centaur-chess-shows-power_b_6383606 (accessed Apr 5, 2019). 
(58) Sahigara, F. Comparison of Different Approaches to Define the Applicability Domain of QSAR Models. Molecules 2012, 17, 4791-4810.

(59) Sheridan, R. P. Using Random Forest To Model the Domain Applicability of Another Random Forest Model. J. Chem. Inf. Model. 2013, 53 (11), 2837-2850. https://doi.org/10.1021/ci400482e.

(60) Liu, R.; Wallqvist, A. Molecular Similarity-Based Domain Applicability Metric Efficiently Identifies Out-of-Domain Compounds. J. Chem. Inf. Model. 2019, 59 (1), 181-189. https://doi.org/10.1021/acs.jcim.8b00597.

(61) Liu, R.; Wang, H.; Glover, K. P.; Feasel, M. G.; Wallqvist, A. Dissecting Machine-Learning Prediction of Molecular Activity: Is an Applicability Domain Needed for Quantitative Structure-Activity Relationship Models Based on Deep Neural Networks? J. Chem. Inf. Model. 2019, 59 (1), 117-126. https://doi.org/10.1021/acs.jcim.8b00348.

(62) Liu, R.; Glover, K. P.; Feasel, M. G.; Wallqvist, A. General Approach to Estimate Error Bars for Quantitative Structure-Activity Relationship Predictions of Molecular Activity. J. Chem. Inf. Model. 2018, 58 (8), 1561-1575. https://doi.org/10.1021/acs.jcim.8b00114.

(63) Mehta, M. Biopharmaceutics Classification System (Bcs).; John Wiley \& Sons, 2017.

(64) Silver, D.; Schrittwieser, J.; Simonyan, K.; Antonoglou, I.; Huang, A.; Guez, A.; Hubert, T.; Baker, L.; Lai, M.; Bolton, A.; Chen, Y.; Lillicrap, T.; Hui, F.; Sifre, L.; van den Driessche, G.; Graepel, T.; Hassabis, D. Mastering the Game of Go without Human Knowledge. Nature 2017, 550 (7676), 354-359. https://doi.org/10.1038/nature24270.

(65) Galemmo, R. A.; Janssens, F. E.; Lewi, P. J.; Maryanoff, B. E. Memorial Issue in Honor of Dr. Paul A. J. Janssen Preface. J. Med. Chem. 2005, 48 (6), 1685-1685. https://doi.org/10.1021/jm040193r. 
(66) Schein, E. H. Organizational Culture and Leadership, Chapter 10: How Leaders Embed and Transmit Culture, 5th Edition.; Wiley: Hoboken, New Jersey, 2017.

(67) Robb, G. R.; McKerrecher, D.; Newcombe, N. J.; Waring, M. J. A Chemistry Wiki to Facilitate and Enhance Compound Design in Drug Discovery. Drug Discovery Today 2013, 18 (3-4), 141-147. https://doi.org/10.1016/j.drudis.2012.09.002.

(68) Schneider, G. Automating Drug Discovery. Nature Reviews Drug Discovery 2017, 17 (2), 97-113. https://doi.org/10.1038/nrd.2017.232. 
Table of Contents graphic

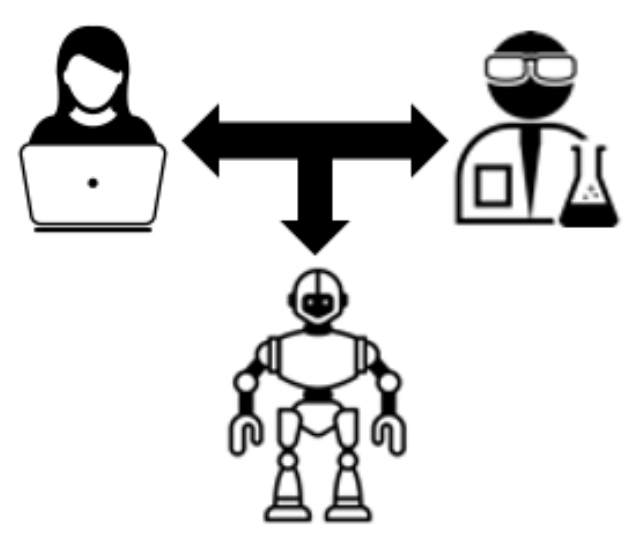

